Tohoku J. Exp. Med., 1989, 159, 313-321

\title{
Production and Characterization of Monoclonal Antibodies against Trypanosoma cruzi-Associated Antigens
}

\author{
Naemi Takasu, Takashi Masuko, Kunio Sugahara and \\ Yoshiyuki Hashimoto \\ Department of Hygienic Chemistry, Pharmaceutical \\ Institute, Tohoku University, Sendai 980
}

\begin{abstract}
Takasu, N., Masuko, T., Sugahara, K. and Hashimoto, Y. Production and Characterization of Monoclonal Antibodies against Trypanosoma cruzi-Associated Antigens. Tohoku J. Exp. Med., 1989, 159 (4), 313-321 — Nine monoclonal antibodies $(\mathrm{mAb})$ recognizing characteristic antigens in Trypanosoma $(T$.) cruzi were obtained from hybridomas which had been established by a fusion between mouse myeloma cells and the spleen cells of a mouse immune to the epimastigote form of $T$. cruzi (Tulahuen strain). Antigen specificities of these $\mathrm{mAb}$ were assessed by an indirect immunofluorescence (IIF) method using T. cruzi in different life cycles (amastigote and trypomastigote) and other members of the Trypanosomatidae. The mAb were classified into 3 groups from their reaction patterns to different parasites: 1) The strain specific $\mathrm{mAb}$ that reacted only with $T$. cruzi Tulahuen epimastigote, 2) the species specific $\mathrm{mAb}$ that reacted with all $T$. cruzi strains but not with other species of parasites, and 3) the mAb that were cross-reactive with other species of Trypanosomatidae. Most mAb were specific to epimastigote form of $T$. cruzi, but some reacted weakly with trypomastigote and amastigote form of the parasites. Immunoblotting and glycolipid analyses of the membrane fraction of homogenized parasites using the $\mathrm{mAb}$ identified at least 3 distinct antigenic molecules; those of protein nature having Mr. 43,000 and 58,000 and Mr. 43,000 and 62,000 and molecule(s) of glycolipid nature.

Trypanosomas : Trypanosoma antigens; monoclonal antibodies
\end{abstract}

Chagas' disease caused by Trypanosoma cruzi ( $T$. cruzi) infection arises a serious problem in Latin America. Ten to twenty million people are believed infected and present chemotherapy is unsatisfactory (Gutteridge 1985). T. cruzi has a complex life cycle and exists in at least four distinct morphological forms : Epimastigotes and an infective form of metacyclic trypomastigotes are found as the insect vector, and trypomastigotes are present in the blood stream of vertebrate hosts, while amastigotes live as intracellular parasites (Brener 1973). The evidence for antigenic differences between the different stages of the parasite lifecycle as well as antigenic differences between strains and cloned stocks of T. cruzi have been reported (Araujo and Remington 1981; Bongertz and Dvorak 1983 ; Chapman et al. 1984).

Received November 7, 1989; revision accepted for publication November 27, 1989. 
A major cell-surface antigen detected by sera from patients with Chagas' disease is a $90 \mathrm{kDa}$ glycoprotein (Nogueira et al. 1981; Snary 1983). This glycoprotein has been found on epimastigotes, amastigotes and blood trypomastigotes. A $72 \mathrm{kDa}$ glycoprotein specific for the insect stages of the life cycle of $T$. cruzi has been identified by cell-surface labelling and mAb immunoprecipitation studies using anti-parasite antibodies (Nogueira et al. 1981; Sher et al. 1983). A $25 \mathrm{kDa}$ glycoprotein has been isolated from soluble extracts of epimastigotes. This glycoprotein, which is also present in trypomastigotes, reacts strongly with sera from infected animals, and, despite being isolated as a soluble component, is associated with the surface membrane of the parasite (Scharfstein et al. 1983).

Although a variety of monoclonal antibodies have been produced against this parasite, the biochemical properties or biological significance of the antigens are yet unclear, as compared with antigens of mammalian cells. In order to understand the nature of $T$. cruzi-associated antigens and to undertake diagnostic or therapeutic applications, we have tried to produce monoclonal antibodies specific to this parasite.

\section{Materials and Methods}

Parasites

The Tulahuen, Y, and Berenice strains of T. cruzi, T. gambiense, Leishmania donovani and Leishmania braziliensis used in this study were donated by Dr. Y. Kaneda, the Department of Parasitology, School of Medicine, Tokai University, Kanagawa, Japan.

The epimastigote form of $T$. cruzi and promastigote form of Leishmania were grown in liver infusion tryptose (LIT) medium containing $5 \%$ calf serum at $28^{\circ} \mathrm{C}$. Trypomatigotes and amastigotes of $T$. cruzi Tulahuen were obtained from cultures of HeLa cells infected with the parasites. Trypomastigotes of $T$. gambiense were obtained from the peripheral blood of infected mice, and washed 3 times with PBS after treatment with $(0.83 \%)$ ammonium chloride solution.

P3x63Ag8.653 mouse myeloma cells were grown in RPMI 1640 medium containing $10 \%$ $\mathrm{FCS}$ at $37^{\circ} \mathrm{C}$, and used for cell fusion.

\section{Immunization of mice}

T. cruzi epimastigotes $\left(5 \times 10^{7}\right.$ cells) were washed 4 times with PBS and suspended in $1 \mathrm{ml}$ PBS. After killing by freezing in liquid nitrogen, the parasites were mixed with an equal volume of Freund's complete adjuvant (Difco Laboratories, Detroit, MI, USA) for the first immunization or Freund's incomplete adjuvant (Difco Laboratories) for the booster injections. $\quad \mathrm{BALB} / \mathrm{c}$ mice of 6 weeks of age were immunized first by an s.c. injection and then received two booster i.p. injections of the parasite preparation. The final booster immunization was performed by i.p. and i.v. injections of the parasites without adjuvant.

\section{Preparation of hybridomas}

Hybridomas were prepared accoring to the method of Masuko (Masuko et al. 1985). In brief, three days after the final immunization, spleen cells of an immunized mouse were fused with P3x63Ag8.653 mouse myeloma cells in the presence of polyethylene glycol 1540. The cells were then suspended in RPMI 1640 medium containing $10 \%$ FCS and distributed into Falcon 96-well plates. One day after the fusion, hybridomas were selected by culture of the cells in medium containing hypoxanthine, aminopterin and thymidine (HAT medium). 
Reactivity of antibodies in culture of hybridomas was checked by the enzyme-linked immunosorbent assay (ELISA) and the indirect immunofluorescence assay (IIF). Selected hybridomas were cloned by the limiting dilution method using mouse thymocytes as the feeder cells. Cloned hybridoma cells were injected i.p. into syngeneic mice which had been treated with pristane (Aldrich Chemical Co., Milwaukee, WI, USA).

\section{$I I F$}

IIF was performed by means of a micro-immunofluorescence assay developed in our laboratory. (Detail for the method is reported in an accompanied paper; Takasu et al. 1989). T. cruzi and others parasites were washed twice with PBS and suspended in PBS at a concentration of $5 \times 10^{5}$ parasites $/ \mathrm{ml}$. Aliquots $(10 \mu \mathrm{l})$ of the suspension were distributed in wells of a Nunc plate (Terasaki's plate) and centrifuged at $1,800 \times g$ for $10 \mathrm{~min}$. After removing the supernatants, the parasites in wells were air-dried, fixed with cold acetonmethanol ( $1: 1)$ and treated with $\mathrm{mAb}$ (hybridoma culture supernatants) for $30 \mathrm{~min}$ at $37^{\circ} \mathrm{C}$, washed with PBS for 3 times, and then treated with fluoresceineisothiocyanate (FITC)-conjugated rabbit anti-mouse immunoglobulins. After 3-washings with PBS, the samples were observed under an Olympus fluorescence microscope.

\section{Isolation of parasite membranes}

$T$. cruzi epimastigotes were collected from culture by centrifugation at $750 \times g$ for 10 min at $4^{\circ} \mathrm{C}$ and washed 4 times with PBS. PBS containing $\operatorname{lmM}$ phenylmethylsulfonyl fluoride was added to the cell pellet and the suspension was sonicated for $30 \mathrm{~min}$. After centrifugation at $500 \times g$ for $15 \mathrm{~min}$ at $4^{\circ} \mathrm{C}$, the supernatants were further centrifuged at $105,000 \times g$ for $60 \mathrm{~min}$. The resultant precipitates were used as a crude membrane fraction.

\section{ELISA}

Aliquots $(50 \mu 1)$ of a suspension of parasite membranes in PBS (equivalent to $10^{6}$ parasites) were added to wells of polyvinyl chloride plates and incubated overnight at $4^{\circ} \mathrm{C}$ to permit the passive adsorption of the antigen to the plate. The plates were washed once with PBS and blocked with each $100 \mu \mathrm{l}$ of PBS containing $1 \%$ bovine serum albumin (BSA) for $1 \mathrm{hr}$. Following incubation with $50 \mu \mathrm{l}$ of hybridoma culture supernatants for 1 $\mathrm{hr}$, the plates were washed 3 times with PBS, and then $50 \mu 1$ of $1: 200$ diluted rabbit anti-mouse immunoglobulins (Dako, Glostrup, Denmark) in PBS containing 1\% BSA was added to each well. After 1 -hr incubation and 3-washings with PBS, $50 \mu \mathrm{l}$ of $1: 1,000$ diluted horseradish peroxidase-conjugated protein A (HRP) (Zymed Laboratory Inc., San Francisco, CA, USA) was added to wells and incubated for $1 \mathrm{hr}$. After washing, residual HRP in wells was assayed by developing the color with $100 \mu \mathrm{l}$ of $0.05 \% 2,2^{\prime}$-azinobis (3-ethylbenzthiazoline sulfonic acid) (ABTS) and $0.01 \%$ hydrogen peroxide and measuring absorbance at $414 \mathrm{~nm}$ using an InterMed NJ-2000 Immunoreader.

\section{Extraction of glycolipids from $T$. cruzi epimastigotes}

Epimastigotes $\left(3 \times 10^{8}\right.$ cells) were collected from a liver infusion tryptose (LIT) culture and washed three times with saline. Glycolipids in the parasites were extracted by treating them ultrasonically in $10 \mathrm{ml}$ of chloroform-methanol $(2: 1)$ (Saito and Hakomori 1971). The resulting extracts were used as antigens for ELISA, where the extracts corresponding to $10^{7}$ parasites were distributed to each well of an ELISA plate and assayed an amount of antigen recognized by $\mathrm{mAb}$.

\section{Immunoblotting}

$T$. cruzi epimastigote membranes were solubilized in sample buffer containing $2 \%$ sodium dodecyl sulfate (SDS) and subjected to polyacrylamide-gel electrophoresis (PAGE) in slab gels of $1.5 \mathrm{~mm}$ thick according to the method of Laemmli (1970). Separated proteins were transferred to nitrocellulose paper according the method of Towbin (Towbin et al. 
1979), and assayed by ELISA for the immunoreactivity against anti-parasite mAb.

\section{RESULTS}

\section{Hybridomas}

Following screening of culture supernatants by IIF and ELISA, it was confirmed that many hybridomas produced antibodies against $T$. cruzi epimastigotes. Nine hybridoma cultures were selected for their immunoreactivities to trypomastigotes and amastigotes of $T$. cruzi Tulahuen, $\mathrm{Y}$ and Berenice strains and cloned by the limiting dilution method. Monoclonal antibodies produced by these nine cloned hybridomas were named ACE-1, -2, -3, -4, -5, -6, -7, -8 and -9 . Immunoglobulin isotype of ACE-1, $-2,-3$ and -5 was of IgM and that of ACE-4, $-6,-7,-8$ and -9 was of IgG1, and all contained $\mathrm{k}$ light chains.

\section{Antigen specificity of $m A b$}

The antigen specificity of mAb was analyzed by IIF using the acetone-fixed $T$. cruzi epimastigotes, amastigotes, and trypomastigotes as target cells. The results are summarized in Table 1 . All showed positive reaction against epimastigotes. ACE-3, -4, -5 and -6 also reacted with amastigotes, and ACE-7 and -9 with trypomastigotes. Reactivity of the $\mathrm{mAb}$ with amastigotes and trypomastigotes was weak, as compared with their reactivity with epimastigotes.

Table 1 also shows the reactivity of the mAb with the epimastigote form of T. cruzi $\mathrm{Y}$ and Berenice strains, other members of the Trypanosomatidae such as $T$. gambiense, L. donovani and L. brazilienses, and HeLa cells (human cervix carcinoma). ACE-1, -2, -3 reacted only with $T$. cruzi Tulahuen among the parasites tested but ACE-2 also reacted strongly with HeLa cells. ACE-4, -5 and

TABLE 1. Reactivity of anti-T. cruzi mAb against various parasites and HeLa cells

\begin{tabular}{|c|c|c|c|c|c|c|c|c|c|}
\hline \multirow{2}{*}{ Parasites } & \multicolumn{9}{|c|}{ mAb (ACE-) } \\
\hline & 1 & 2 & 3 & 4 & 5 & 6 & 7 & 8 & 9 \\
\hline \multicolumn{10}{|l|}{ T. cruzi Tulahuen } \\
\hline Epimastigote & H & \# & \# & H & H & H & 世 & \# & \# \\
\hline Trypomastigote & - & - & - & - & - & - & + & - & + \\
\hline Amastigote & - & - & + & + & + & + & - & - & - \\
\hline$T$. cruzi Berenice & - & - & - & - & - & - & H & H & H \\
\hline T. $c z u z i \mathrm{Y}$ & - & - & - & + & + & + & H & - & H \\
\hline T. gambiense & - & - & - & - & - & - & - & + & + \\
\hline L. braziliense & - & - & - & - & - & - & - & - & - \\
\hline L. donovani & - & - & - & - & - & - & - & - & - \\
\hline HeLa (human) & - & H & - & - & - & - & - & - & - \\
\hline
\end{tabular}

Acetone-fixed parasites or HeLa cells were analyzed for their reactivity with $\mathrm{mAb}$ by IIF as described in Materials and Methods. 

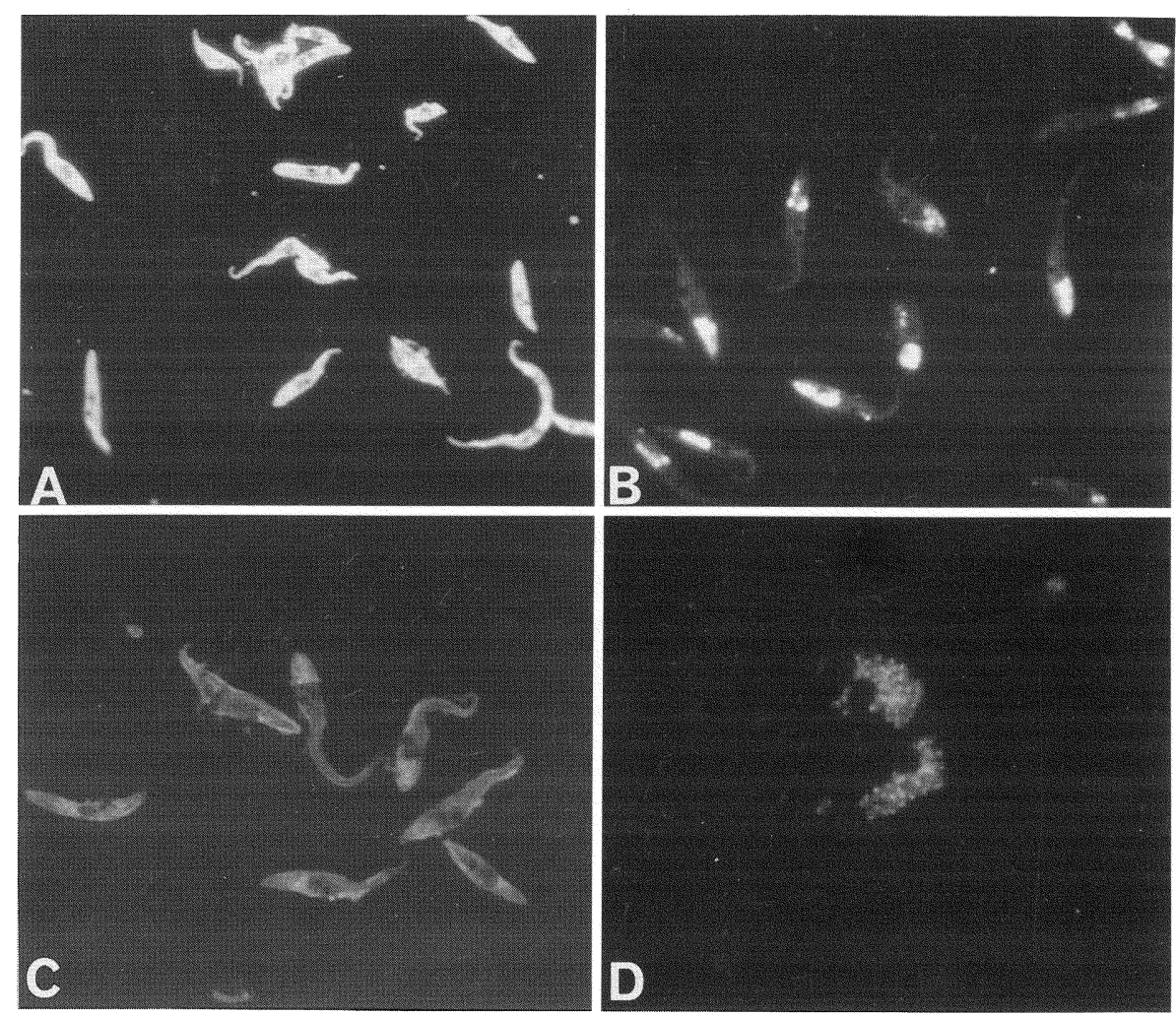

Fig. 1. Immunofluorescence pattern of acetone-fixed parasites stained with antiT. cruzi mAb. Acetone-fixed parasites were stained with mAb and FITClabed rabbit anti-mouse immunoglobulins. A-C, epimastigote form of $T$. cruzi. A, ACE-8 mAb; B, ACE-9 mAb; C, ACE-3 mAb. D, Amastigote form of $T$. cruzi in HeLa cells stained with ACE-4 mAb.

-6 reacted with T. cruzi Y strain, ACE-8 with Berenice strain, and ACE-7, and -9 with both $T$. cruzi $\mathrm{Y}$ and Berenice strains. The IIF staining in an epimastigote form of parasites fixed in cold acetone revealed bright stain over the entire organism (ACE-2, $-6,-7$, and -8 ) or bright stain of superior part of the parasites (ACE-1, -4, -5 and -9). ACE-3 stained partially the superior and flagellum parts of the parasites (Fig. 1). ACE-4 also stained an amastigote form of $T$. cruzi resided in HeLa cells.

The reactivity of living parasites was positive for ACE-2 and ACE-5, where the parasites agglutinated with the $\mathrm{mAb}$ and were immunofluorescently stained as clots (data not shown).

\section{Biochemical characterization of $T$. cruzi-associated antigens defined by $m A b$}

To assess biochemical characters of antigens recognized by $\mathrm{mAb}$, reactivities of the membrane fractions of parasites with mAb were assayed by ELISA. As 
TABLE 2. Reactivity of anti-T. cruzi mAb against T. cruzi epimastigote membranes, by ELISA

\begin{tabular}{ccccccccc}
\hline \multicolumn{10}{c}{ Absorbance (at } & $414 \mathrm{~nm}$ ) with $\mathrm{mAb}$ (ACE-) \\
1 & 2 & 3 & 4 & 5 & 6 & 7 & 8 & 9 \\
\hline 0.99 & 0.99 & 0.54 & 0.92 & 1.04 & 1.20 & 1.76 & 0.64 & 1.04 \\
\hline
\end{tabular}

shown in Table 2, all $\mathrm{mAb}$ reacted with the membrane fractions. We then assessed the molecular weight of the mAb-defined antigens by SDS-PAGE and Western blotting. The results are depicted in Fig. 2. ACE-8 detected the components of apparent molecular weights of 43,000 and 58,000 from T. cruzi Tulahuen epimastigote, but it did not react with the components of $T$. cruzi $\mathrm{Y}$. ACE-9 detected the components of molecular weights of about 43,000 and 62,000 from both $T$. cruzi Tulahuen and $T$. cruzi $\mathrm{Y}$.

Reactivity of the nine mAb with epimastigote glycolipids are shown in Fig. 3. ACE-1, $-4,-5,-7$ and -9 reacted with the glycolipids fraction extracted from a epimastigote form of $T$. cruzi but other $\mathrm{mAb}$ did not react with the glycolipids.

A



B

ACE-9



Fig. 2. Molecular weights of the mAb-defined antigens determined by SDS-PAGE and Western blotting. Molecular weights of standard materials are shown as K, kilo dalton. A, ACE-8 mAb ; B, ACE-9; T, T. cruzi Tulahuen; Y, T. cruzi Y. 


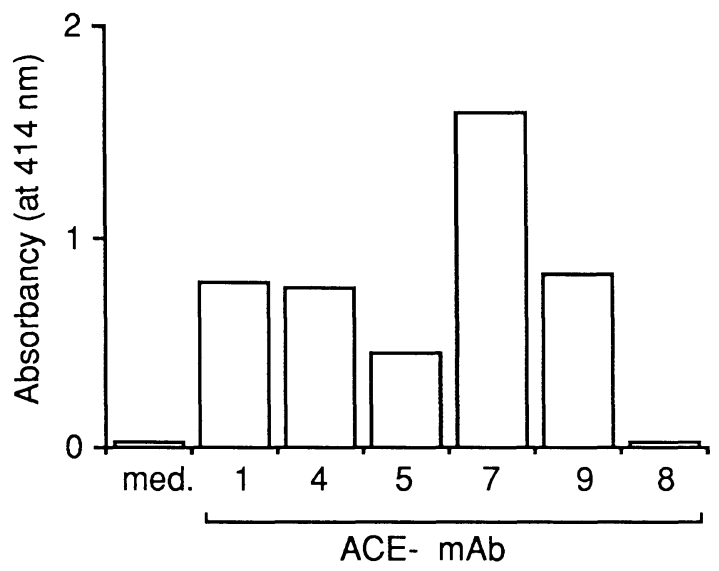

Fig. 3. Reactivity of $\mathrm{mAb}$ with a glycolipid fraction extracted from epimastigote form of T. cruzi. Glycolipid fractions were extracted from the parasites and their reactivity with anti-T. cruzi mAb was assayed by ELISA.

\section{Discussion}

In this work, we have produced nine $\mathrm{mAb}$ against the epimastigote form of $T$. cruzi Tulahuen and characterized the mAb-defined antigens present in $T$. cruzi and the related parasites. Based on the reactivity with various parasites, the $\mathrm{mAb}$ are classified to 3 groups ; 1) specific to the epimastigote form of $T$. cruzi Tulahuen (ACE-1, -2 and -3) ; 2) selective to parasites of $T$. cruzi species (ACE-4, $-5,-6$ and -7$)$; and 3 ) cross-reactive with other species of parasites such as $T$. gambiense (ACE-8 and -9).

As for the biochemical character of parasite antigens defined by the $\mathrm{mAb}$, two $\mathrm{mAb}$ definitely reacted with glycoprotein antigens having Mr. of 43,000 and 62,000 (ACE-9), and Mr. 43,000 and 58,000 (ACE-8). An epitope recognized by ACE-9 $\mathrm{mAb}$ appeared to be present on a sugar(s), because this $\mathrm{mAb}$ reacted not only with the glycoprotein antigens but also reacted with glycolipid(s) extracted from $T$. cruzi.

MAb to $T$. cruzi-associated antigens have also been prepared in other laboratories and molecular weight of the antigens recognized by these $\mathrm{mAb}$ are reported ; 75,82 and $90 \mathrm{kDa}$ antigens present in metacyclic trypomastigotes (Teixeira and Yoshida 1986) ; $85 \mathrm{kDa}$ antigen present in trypomastigotes (Alves et al. 1986) ; 40 $\mathrm{kDa}$ antigen (Segura et al. 1986), $43 \mathrm{kDa}, 36 / 52 \mathrm{kDa}$ and $32 / 50 \mathrm{kDa}$ antigens (Tachibana and Kaneda 1984), and $72 \mathrm{kDa}$ antigen (Sher et al. 1983) present in epimastigotes ; $25 \mathrm{kDa}$ antigen (Scharsfstein et al. 1983), $24 \mathrm{kDa}, 43 \mathrm{kDa}$ and 51 $\mathrm{kDa}$ antigens (Orozco et al. 1984), 58/60 and $68 \mathrm{kDa}$ antigens (Manas et al. 1986) and 85 and $90 \mathrm{kDa}$ antigens (Segura et al. 1986) present in multiple forms of $T$. cruzi. Considering from the molecular weight of antigens, the $\mathrm{mAb}$ from other laboratories and several of our $\mathrm{mAb}$ may define the same or similar parasite 
antigens. However, different panels of parasites have been used in the assays for antigens, except the experimental system of, Tachibana and Kaneda (1984). Therefore, it is unclear yet whether our and other $\mathrm{mAb}$ recognize the same antigens or not.

In contrast to studies on protein antigens of $T$. cruzi, the informations about glycolipid structure of this parasite are extremely scarce. We examined the reactivities of $\mathrm{mAb}$ against the glycolipid fraction extracted from the parasites, since $\mathrm{mAb}$ reactive to glycolipids components of $T$. cruzi have not been reported. We found that ACE-1, $-4,-5,-7$ and -9 reacted with the glycolipid fraction from T. cruzi.

As for the subcellular localization of the mAb-defined antigens, the antigens mainly distributed in intracellular components of parasites (see Fig. 1). However, ACE-2 and -5 appeared to recognize also the cell-surface antigens of $T$. cruzi because they could agglutinate unfixed living parasites and showed cytotoxicity to $T$. cruzi in the presence of rabbit complement (data not shown).

$\mathrm{MAb}$ prepared in this work will be useful for classification of trypanosomas.

\section{Acknowledgments}

We thank Drs. Y. Kaneda, Y. Tachibana and K. Nagakura, Tokai University for their kind supply of parasites and for their helpful advises.

\section{References}

1) Alves, M.J.M., Abuin, G., Kuwajima, V.Y. \& Colli, W. (1986) Partial inhibition of trypomastigote entry into cultured mammalian cells by monoclonal antibodies against a surface glycoprotein of Trypanosoma cruzi. Mol. Biochem. Parasitol., 21, 75-82.

2) Araujo, F.G. \& Remington, J.S. (1981) Characterization of stages and strains of Trypanosoma cruzi by analysis of cell membrane components. J. Immunol., 127, 855-859.

3) Bongertz, V. \& Dvorak, J.A. (1983) Trypanosoma cruzi: Antigenic analysis of cloned stocks. Am. J. Trop. Med. Hyg., 32, 716-722.

4) Brener, Z. (1973) Biology of Trypanosoma cruzi. Ann. Rev. Microbiol., 27, 347382.

5) Chapman, M.D., Snary, D. \& Miles, M.A. (1984) Quantitative differences in the expression of a 72,000. Molecular weight cell surface glycoprotein in Trypanosoma cruzi Zymodemes. J. Immunol., 132, 3149-3153.

6) Gutteridge, W.E. (1985) Existing chemotherapy and its limitations. Br. Med. Bull., 41, 162-168

7) Laemmli, U.K (1970) Clevage of structural proteins during the assembly of the head of bacteriophage T4. Nature, 227, 680-685.

8) Manas, I., Lozano, J., Campos, M., Gonzalez, J., Ruiz-Cabello, F. \& Garrido, F. (1986) Production of monoclonal antibodies to metacyclic trypomastigotes of Trypanosoma cruzi. Hybridoma, 5, 147-154.

9) Masuko, T., Abe, J., Yagita, H. \& Hashimoto, Y. (1985) Human bladder cancer cell-surface antigens recognized by murine monoclonal antibodies raised against T24 bladder cancer cells. Jpn. J. Cancer Res. (Gann), 76, 386-394.

10) Nogueira, N., Chaplan, S., Tydings, J.D., Unkelless, J. \& Cohn, Z. (1981) Trypanosoma cruzi. Surface antigens of blood and culture forms. J. Exp. Med., 
153, 629-639.

11) Orozco, O., Afchain, D., Dissous, C., Rodriguez, C., Ovlaque, G., Lemesre, J.L., Loyens, M. \& Capron, A. (1984) Different monoclonal antibodies against the component 5 specific for Trypanosoma cruzi. Am. J. Med. Hyg., 33, 560-568.

12) Saito, T. \& Hakomori, S. (1971) Quantitative isolation of total glycosphingolipids from animals cells. J. Lipid Res., 12, 257-259.

13) Scharfstein, J., Rodrigues, M.M., Alves, C.A., De Souza, W., Previato, J.O. \& Mendonaca, P.L. (1983) Trypanosoma cruzi: Description of a highy surface antigen defined by human antibodies. J. Immunol., 131, 972-976.

14) Segura, E.L., Bua, J., Rosestein, C.A., Subias, E., Esteva, M., Moreno, M. \& Ruiz, A.M. (1986) Monoclonal antibodies against the flagellar fraction of epimastigotes of Trypanosoma cruzi: Complement-mediated lytic activity against trypomasigotes and passive immunoprotection in mice. Immunol. Lett., 13, 165-171.

15) Sher, A., Crane, M.S.T.J. \& Kirchhoff, L.V. (1983) Incubation in mice provides a signal for the differentiation of Trypanosoma cruzi epimastigotes to trypomastigotes. J. Protozool., 30, 278-283.

16) Snary, D. (1983) Cells surface glycoproteins of Trypanosoma cruzi; protective immunity in mice and antibody levels in human chagasic sera. Trans. R. Soc. Trop. Med. Hyg., 77, 126-129.

17) Tachibana, H. \& Kaneda, Y. (1984). Monoclonal antibodies to Trypanosoma cruzi: Characterization of specific antigens in epimastigote stage. Jpn. J. Parasitol., 33, 457-466.

18) Takasu, N., Masuko, T., Hojo, H. \& Hashimoto, Y. (1989) A microtestplateimmunofluorescence assay for anti-Trypanosoma cruzi antibodies. Tohoku J. Exp. Med., 159, 307-312.

19) Teixeira, M.M.G. \& Yoshida, N. (1986) Stage-specific surface antigens of metacyclic trypomastigotes of Trypanosoma cruzi identified by monoclonal antibodies. Mol. Biochem. Parasitol., 18, 271-282.

20) Towbin, H., Staehelin, T. \& Gordon, J. (1979) Electrophoretic transfer and some aplications. Proc. Natl. Acad. Sci. USA, 76, 4350-4354. 